\title{
DRO1 sensitizes colorectal cancer cells to receptor-mediated apoptosis
}

\author{
ANDREAS HERBST, CONSTANZE BAYER, CLAUDIA WYPIOR and FRANK T. KOLLIGS
}

Second Department of Medicine, University of Munich, Munich, Germany

Received March 29, 2011; Accepted June 21, 2011

DOI: $10.3892 / \mathrm{ol} .2011 .356$

\begin{abstract}
The molecule DRO1 (down-regulated by oncogenes 1) is a potential tumor suppressor protein that is frequently down-regulated in primary colorectal cancers and colorectal cancer cell lines. Although the mechanism of DRO1 action has yet to be elucidated, previous data have suggested that DRO1 interferes with tumor growth by sensitizing cells to apoptosis. The effect of DRO1 expression on receptor-, mitochondrial- and endoplasmic reticulum-mediated apoptosis in colorectal cancer cell lines was analyzed in this study, following the generation of DLD1/DRO1 and HCT116/ DRO1 cell lines. Cells were cultured, and then analyzed using flow cytometry. DRO1 was found to sensitize cells to receptor-mediated apoptosis by promoting the activation of components of the death-inducing signaling complex (DISC).
\end{abstract}

\section{Introduction}

Colorectal cancer is the third most common type of cancer and a major cause of cancer-related morbidity. Deregulation of $\beta$-catenin due to mutations in the $\mathrm{Wnt} / \beta$-catenin signaling pathway is an early event in colorectal carcinogenesis, leading to the majority of sporadic colorectal cancers. Among the genes regulated by $\beta$-catenin, we identified the gene CCDC80 encoding the protein DRO1 (down-regulated by oncogenes 1), whose expression is frequently down-regulated in primary colorectal cancers and colorectal cancer cell lines (1). The DRO1 protein sequence comparison revealed the presence of a coiled-coil domain and three other domains [internal repeats (IRs) 1-3]. The IR domains 1-3 have a high homology to a domain in a protein called DRS (down-regulated by src) (1). DRS is involved in the regulation of endoplasmic reticulum (ER)-mediated apoptosis, suggesting that DRO1 plays a role in the regulation of apoptosis (2). Constitutive expression of DRO1 in HCT116 colorectal cancer cells sensitized these

Correspondence to: Dr Frank T. Kolligs, Second Department of Medicine, University of Munich, Marchioninistrasse 15, 81377 Munich, Germany

E-mail: fkolligs@med.uni-muenchen.de

Key words: colorectal cancer, receptor-mediated apoptosis, DRO1, DISC, FADD cells to receptor-mediated apoptosis induced by activation of the death receptor CD95 (1). Binding of the CD95 ligand to its cognate receptor results in the formation of the death-inducing signaling complex (DISC), which comprises caspase- 8 and the adapter molecule FADD. Upon DISC formation, caspase- 8 is converted from its inactive pre-form to the active form and promotes apoptosis by cleaving, thereby activating downstream effector caspases including caspase-1, $-3,-6$ and -7 (3). Apart from receptor-mediated apoptosis (or the 'extrinsic apoptosis pathway'), activation of the 'intrinsic apoptosis pathway', involving permeabilization of the outer mitochondrial membrane (MOMP), or ER-mediated apoptosis due to ER stress is capable of inducing apoptosis (4-6). The down-regulation of DRO1 expression in colorectal cancer cells and its role in sensitizing cells to CD95-dependent apoptosis suggests that DRO1 is a potential tumor suppressor protein that plays a crucial role in colorectal carcinogenesis. This hypothesis is further supported by the observation that transformation of the rat epithelial cell line RK3E with various oncogenes resulted in the loss of DRO1 expression, while the re-expression of DRO1 in HCT116, BxPc3 and transformed RK3E cells prevented the formation of colonies in soft agar assays (1).

The aim of this study was to investigate whether the function of DRO1 is limited to CD95-mediated apoptosis or whether DRO1 was capable of sensitizing cells for TRAILand TNF-mediated extrinsic apoptosis as well as intrinsic apoptosis and ER-mediated apoptosis. Subsequently, two colorectal cancer cell lines were generated that inducibly expressed DRO1, and the effects of various apoptosis-inducing agents on the survival of these cells following induction of DRO1 expression were studied. This study revealed that expression of DRO1 sensitizes cells for receptor-mediated apoptosis by activating components of the DISC.

\section{Materials and methods}

Plasmids. The inducible episomal expression vector pRTS-1 (7) was digested with the restriction enzyme SfiI, and the hemagglutinin (HA) epitope-tagged coding sequence of DRO1 (Ensembl transcript ID: ENST00000439685) was cloned in this construct, yielding pRTS-1/DRO1.

Cell culture and assays. DLD1 and HCT116 cells were maintained in Dulbecco's modified Eagle's medium (DMEM) 
(PAA, Pascha, Austria) with 10\% FCS (PAA) and pen/strep (PAA). DLD1 and HCT116 cells were stably transfected with the inducible expression construct pRTS-1/DRO1, encoding the open reading frame of DRO1, to yield DLD1/ DRO1 and HCT116/DRO1. For the apoptosis assays, the cells were seeded in $1 \mathrm{ml} \mathrm{DMEM} / 10 \% \mathrm{FCS} /$ pen/strep medium at a density of $8 \times 10^{4}$ cells per well in a 12-well plate (Falcon; Becton-Dickinson, Franklin Lakes, NJ, USA). The following day, cells were stimulated with $1 \mu \mathrm{g} / \mathrm{ml}$ doxycycline to induce DRO1 expression or were left untreated. On day 3, cells were treated with APO [human monoclonal antibody to Fas (Apo 1-3), Alexis, Lörrach, Germany] plus Protein A (recombinant Protein A, BioVision, Ilmenau, Germany), TRAIL (recombinant human TRAIL/TNFSF10, R\&D Systems, Wiesbaden, Germany) and TNF $\alpha$ (recombinant human TNFal TNFSF1A, R\&D Systems), plus $0.2 \mu \mathrm{g}$ actinomycin D (Sigma, Steinheim, Germany), staurosporine (Calbiochem, Darmstadt, Germany), or thapsigargin (Applichem, Darmstadt, Germany) at the indicated concentrations to induce apoptosis. After 16 (TNF $\alpha$ only) or $24 \mathrm{~h}$, attached and floating cells were harvested and stained with propidium iodide, and the number of apoptotic cells was determined by flow cytometry.

Immunodetection. To analyze DRO1 expression, DLD1/ DRO1 and HCT116/DRO1 cells were stimulated with $1 \mu \mathrm{g} / \mathrm{ml}$ doxycycline for the indicated periods of time. Cells were harvested and lysed using reporter gene lysis buffer (Promega, Madison, WI, USA). Protein samples were separated on a 10\% SDS-polyacrylamide gel, transferred onto a PVDF membrane (Immobilon-P, Millipore, Billerico, MA, USA) and the expression of HA epitope-tagged DRO1 was analyzed using the HA antibody 3F10 (Roche, Grenzach-Wyhlen, Germany). Expression of caspase- 8 and FADD was analyzed by immunoblotting with the corresponding antibodies (anti-caspase-8, Cell Signaling Technology, Danvers, MA, USA; anti-FADD, Santa Cruz Biotechnology, Santa Cruz, CA, USA) after treating DLD1/DRO1 and HCT116/DRO1 cells with $1 \mu \mathrm{g} /$ $\mathrm{ml}$ doxycyline for $48 \mathrm{~h}$ and $5 \mathrm{ng}$ (DLD1/DRO1) and $125 \mathrm{ng}$ (HCT116/DRO1) of the CD95-activating antibody APO for $24 \mathrm{~h}$. Actin was used as a loading control (actin antibody, MP Bio, Heidelberg, Germany).

\section{Results}

Inducible DRO1 expression in DLDI and HCT116 colorectal cancer cells. Recently, it was reported that constitutive expression of DRO1 sensitizes HCT116 cells for CD95-induced apoptosis (1). To analyze the effect of inducible DRO1 expression on apoptosis, colorectal cancer cell lines DLD1 and HCT116 were stably transfected with the inducible expression vector pRTS-1/DRO1, to generate the cell lines DLD1/DRO1 and HCT116/DRO1. These cell lines were stimulated with doxycycline for different periods of time to induce DRO1 expression. DRO1 expression was detectable in the two cell lines as early as $4 \mathrm{~h}$ after the addition of doxycycline and reached a plateau after $16 \mathrm{~h}$ (Fig. 1A).

DRO1 expression sensitizes cells to receptor-mediated apoptosis. To confirm that the expression of DRO1 sensitizes cells to CD95-dependent apoptosis, DLD1/DRO1 and HCT116/
A

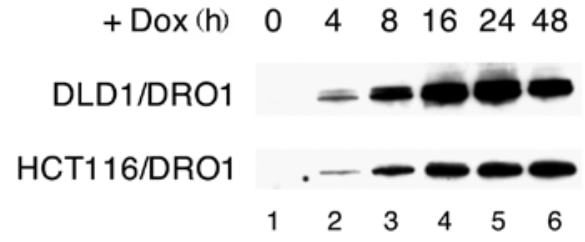

B
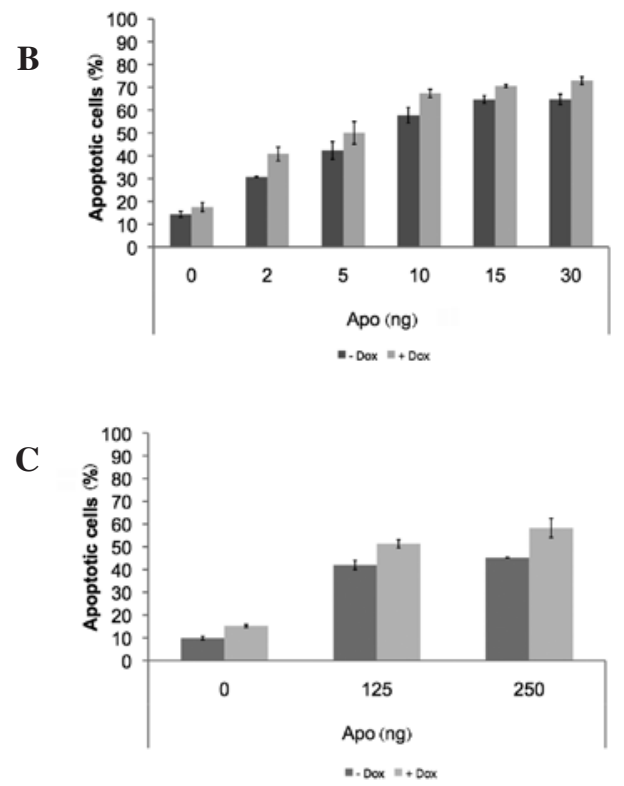

Figure 1. Induced expresssion of DRO1 sensitizes cells for CD95-mediated apoptosis. (A) Colorectal cancer cells DLD1 and HCT116 were stably transfected with a doxycycline (Dox)-inducible expression vector encoding the DRO1 protein to yield DLD1/DRO1 and HCT116/DRO1 cells. Cells were stimulated with Dox for the indicated periods of time. Cells were harvested and lysates were analyzed for DRO1 expression by immunoblotting. (B) DLD1/DRO1 and (C) HCT116/DRO1 cells were stimulated with Dox (or left untreated) and treated with increasing amounts of the CD95-activating antibody APO. Cells were stained using propidium iodide and the number of apoptotic cells was determined using flow cytometry.

A

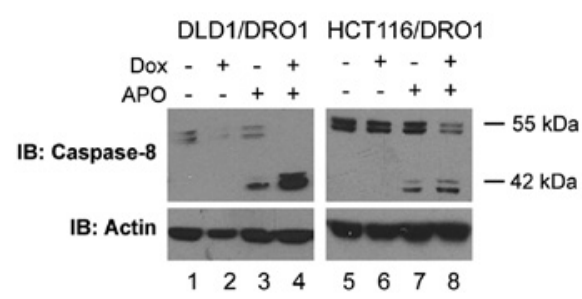

B

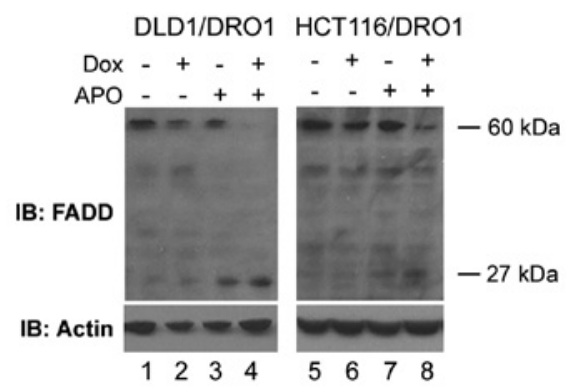

Figure 2. DRO1 increases protein levels of active caspase-8 and FADD. DLD1/DRO1 and HCT116/DRO1 cells were treated with combinations of Dox and APO, and total cell lysates were prepared. Expression of (A) caspase- 8 and (B) FADD protein was analyzed by immunoblotting. Actin was used as a loading control. 
A

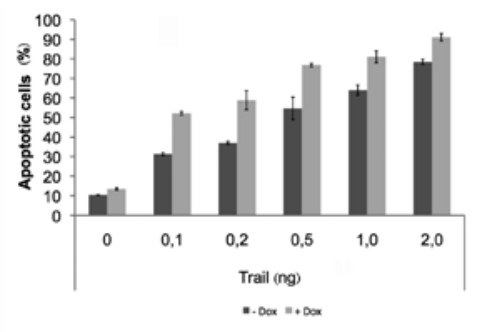

C

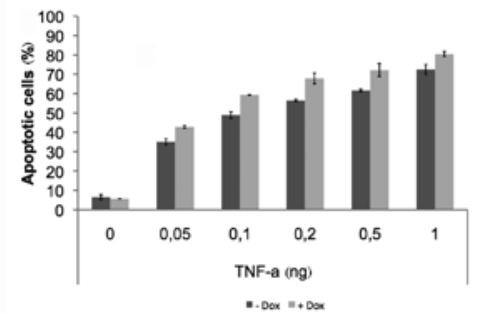

B

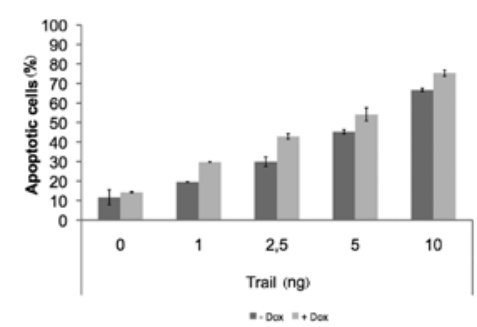

D

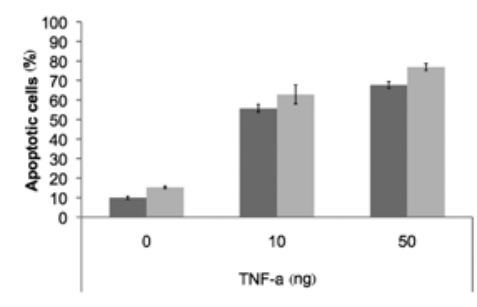

$-.00 x++\infty 0 x$

Figure 3. Expression of DRO1 sensitizes cells for TRAIL- and TNF $\alpha$-induced apoptosis. (A, C) DLD1/DRO1 and (B, D) HCT116/DRO1 cells were stimulated with Dox (or left untreated) and treated with increasing amounts of TRAIL- and TNFa, respectively. Cells were stained using propidium iodide and the number of apoptotic cells was determined using flow cytometry.

A

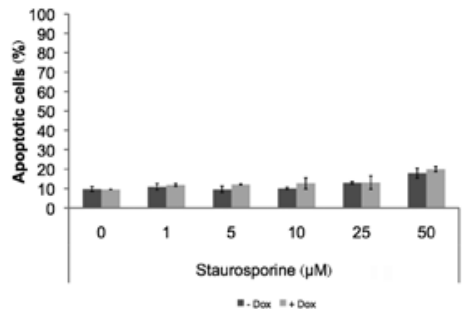

C

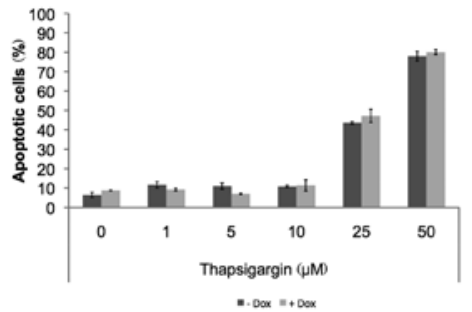

B

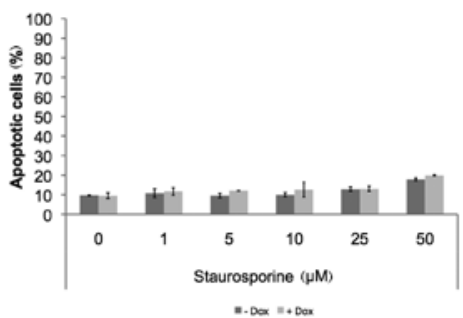

D

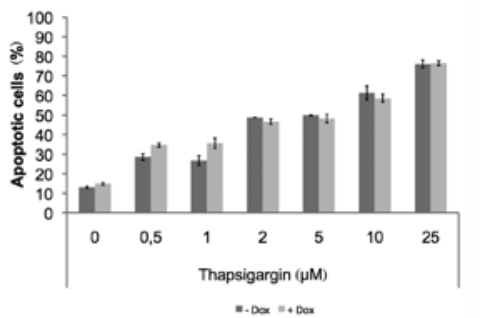

Figure 4. DRO1 does not affect mitochondrial- and ER-mediated apoptosis. (A, C) DLD1/DRO1 and (B, D) HCT116/DRO1 cells were stimulated with Dox (or left untreated) and treated with increasing amounts of staurosporine and thapsigargin, respectively. Cells were stained using propidium iodide and the number of apoptotic cells was determined using flow cytometry.

DRO1 cells were treated with doxycycline and increasing amounts of the CD95-activating antibody APO. In the two cell lines, the number of apoptotic cells was increased by approximately $10 \%$ compared to APO-treated control cells, confirming previous results in constitutively DRO1-expressing HCT116 cells, which revealed that DRO1 is capable of sensitizing cells for receptor-mediated apoptosis (Fig. 1B and C). Since caspase- 8 is a key component of the DISC involved in receptor-mediated apoptosis, we analyzed the manner in which stimulation of DLD1/DRO1 and HCT116/DRO1 cells with the CD95-activating antibody APO and doxycycline affects caspase- 8 activation. Treatment of these cells with APO alone resulted in the activation of caspase- 8 , as measured by the appearance of the active $42 \mathrm{kDa}$ form of caspase- 8 using immunodetection. In these experiments, the simultaneous expression of DRO1 further increased the expression levels of the active caspase- 8 protein (Fig. 2A; compare lanes $3 / 4$ and $7 / 8$, respectively). Apart from caspase-8, the adapter molecule FADD represents a significant component of the DISC complex. To analyze how DRO1 affects the expression of this protein, DLD1/DRO1 and HCT116/DRO1 cells were treated with APO and the appearance of the active $27 \mathrm{kDa}$ form of FADD was studied using immunodetection. Induction of receptor-mediated apoptosis using the antibody APO resulted in an increase of FADD expression in DLD1/DRO1 and HCT116/DRO1 cells. Notably, the co-expression of DRO1 further increased FADD expression in the two cell lines (Fig. 2B), suggesting that DRO1 promotes apoptosis by regulating the activity of FADD.

To determine whether the function of DRO1 is limited to CD95-mediated apoptosis, the cell lines DLD1/DRO1 and HCT116/DRO1 were treated with increasing amounts of TRAIL as well as TNFa. The effects of these reagents 
on the induction of apoptosis in the presence of DRO1 were compared against the effect of the CD95-activating antibody APO. Treatment of DLD1/DRO1 and HCT116/DRO1 cells with TRAIL or TNF $\alpha$ resulted in a concentration-dependent increase in the apoptosis rate. Co-expression of DRO1 increased the TRAIL- and TNF $\alpha$-dependent apoptosis rate by $10-15 \%$ in the two cell lines, similar to the effect of CD95-mediated apoptosis (Fig. 3), indicating that DRO1 plays a role in sensitizing cells for receptor-mediated apoptosis.

DRO1 neither promotes mitochondrial- nor ER-mediated apoptosis. To determine whether DRO1 expression had an effect on the intrinsic apoptotic pathway or ER-mediated apoptosis, the cell lines DLD1/DRO1 and HCT116/DRO1 were treated with increasing concentrations of staurosporine or thapsigargin to induce the intrinsic pathway and ER-induced apoptosis, respectively. In the two cell lines, DRO1 expression did not sensitize cells for apoptosis induced by staurosporine nor thapsigargin (Fig. 4).

\section{Discussion}

Deregulation of $\beta$-catenin activity is a hallmark of sporadic forms of colorectal cancer and occurs early in colorectal carcinogenesis (8). Apart from genes whose expression is induced by $\beta$-catenin, including c-Myc, Sox 9 or HDAC $2, \beta$-catenin also suppresses gene expression. The CCDC80 gene encoding the protein DRO1 belongs to the latter group of genes. An analysis of DRO1 expression revealed that its expression is frequently lost in primary colorectal carcinomas and colorectal cancer cell lines, suggesting that DRO1 expression interferes with tumor progression and that DRO1 may act as a potential tumor suppressor (1). This hypothesis is supported by the observation that transformation of RK3E cells with $\beta$-catenin, $\gamma$-catenin, c-Myc, Ras or Gli results in the loss of DRO1 expression. The re-expression of DRO1 in HCT116, BxPc3 or transformed RK3E cells prevents anchorage-dependent growth in soft agar assays (1). Further analysis revealed that a constitutive expression of DRO1 sensitizes cells to detachment-induced apoptosis (or 'anoikis') and CD95 receptor-mediated apoptosis. By analyzing the activation of components of the DISC, the expression of DRO1 was found to result in an increase of activated caspase- 8 and FADD protein levels when cells were treated with the CD95-activating antibody APO and doxycycline compared to APO alone. These data suggest that DRO1 sensitizes cells to CD95-dependent apoptosis by promoting the formation of DISC. The effect of DRO1 on receptor-mediated apoptosis was not limited to CD95, as the induction of apoptosis using TRAIL and TNF $\alpha$ also reduced the survival of DRO1-expressing cells. However, treatment of DRO1-expressing cells using staurosporine to induce the intrinsic apoptotic pathway or thapsigargin to induce ER-mediated apoptosis did not result in increased cell death. In this respect, DRO1 differs from the protein DRS that is involved in ER-mediated apoptosis, despite the fact that the DRS domain shares a high homology with the IR domains of DRO1 (2).

Although DRO1 expression sensitizes cells for receptor-mediated apoptosis by increasing protein levels of active caspase- 8 and FADD, the precise mechanism remains unclear. The DRO1 protein may support the formation of the DISC or stabilize an already formed DISC, thereby increasing the proteolytic processing of caspase-8 (9). Alternatively, DRO1 may enhance the formation of monomeric active FADD by processing dimeric inactive FADD to promote the recruitment (and subsequent activation) of caspase- 8 into the DISC (10). To elucidate the DRO1 mechanism, we aim to immunoprecipitate and identify DRO1-interacting proteins.

In conclusion, the data clearly show that DRO1 sensitizes colorectal cancer cells for receptor-mediated apoptosis by activating components of DISC, suggesting that this action contributes to the role of DRO1 as a potential tumor suppressor protein.

\section{References}

1. Bommer GT, Jager C, Durr EM, et al: DRO1, a gene down-regulated by oncogenes, mediates growth inhibition in colon and pancreatic cancer cells. J Biol Chem 280: 7962-7975, 2005.

2. Tambe Y, Isono T, Haraguchi S, Yoshioka-Yamashita A, Yutsudo $\mathrm{M}$ and Inoue $\mathrm{H}$ : A novel apoptotic pathway induced by the drs tumor suppressor gene. Oncogene 23: 2977-2987, 2004.

3. Walczak $\mathrm{H}$ and Haas TL: Biochemical analysis of the native TRAIL death-inducing signaling complex. Methods Mol Biol 414: 221-239, 2008.

4. Bergqvist I, Eriksson M, Saarikettu J, Eriksson B, Corneliussen B, Grundstrom T and Holmberg D: The basic helix-loop-helix transcription factor E2-2 is involved in T lymphocyte development. Eur J Immunol 30: 2857-2863, 2000.

5. Chipuk JE and Green DR: How do BCL-2 proteins induce mitochondrial outer membrane permeabilization? Trends Cell Biol 18: 157-164, 2008.

6. Szegezdi E, Macdonald DC, Ni Chonghaile T, Gupta S and Samali A: Bcl-2 family on guard at the ER. Am J Physiol Cell Physiol 296: C941-C953, 2009.

7. Bornkamm GW, Berens C, Kuklik-Roos C, et al: Stringent doxycycline-dependent control of gene activities using an episomal one-vector system. Nucleic Acids Res 33: e137, 2005.

8. MacDonald BT, Tamai K and He X: Wnt/ $\beta$-catenin signaling: components, mechanisms, and diseases. Dev Cell 17: 9-26, 2009.

9. Pennarun B, Meijer A, de Vries EG, Kleibeuker JH, Kruyt F and de Jong S: Playing the DISC: turning on TRAIL death receptor-mediated apoptosis in cancer. Biochim Biophys Acta 1805: $123-140,2010$.

10. Zhang X, Vallabhaneni R, Loughran PA, Shapiro R, Yin XM, Yuan Y and Billiar TR: Changes in FADD levels, distribution, and phosphorylation in $\mathrm{TNF} \alpha$-induced apoptosis in hepatocytes is caspase- 3 , caspase- 8 and BID dependent. Apoptosis 13: 983-992, 2008 . 